\title{
INFLUÊNCIA DA EMBALAGEM DE POLIETILENO DE BAIXA DENSIDADE E DA TEMPERATURA NA CONSERVAÇÃO DO REPOLHO MINIMAMENTE PROCESSADO
}

\section{MARIA M. RINALDI ${ }^{1}$, BENEDITO C. BENEDETTI ${ }^{2}$}

RESUMO: Neste trabalho, foi estudado o efeito da embalagem de polietileno de baixa densidade e da temperatura de armazenagem na conservação de repolho minimamente processado. Os repolhos, previamente resfriados à temperatura de $10^{\circ} \mathrm{C}$, foram submetidos à seleção, lavagem, corte em quatro partes com a retirada do talo central, sanitização, corte em tiras, enxágüe, centrifugação, pesagem e acondicionamento em embalagens plásticas de polietileno de baixa densidade $(70 \mu \mathrm{m})$ e armazenados em câmaras frias nas temperaturas de 1 e $10^{\circ} \mathrm{C}$ por 20 dias. Avaliaram-se dióxido de carbono, oxigênio e etileno na atmosfera interna da embalagem, bem como $\mathrm{pH}$, acidez titulável, sólidos solúveis totais, vitamina $\mathrm{C}$, perda de massa fresca e relação sólidos solúveis totais/acidez no repolho minimamente processado. $\mathrm{O}$ delineamento estatístico utilizado foi o inteiramente casualizado, com três repetições. Os parâmetros de análise, com exceção da vitamina $\mathrm{C}$, perda de massa fresca e etileno, apresentaram variação significativa entre as temperaturas e dias de armazenamento. O repolho armazenado na temperatura de $1^{\circ} \mathrm{C}$ apresentou vida de prateleira ao redor de 15 dias, significativamente maior que a $10^{\circ} \mathrm{C}$. Nesta última, no $8^{-0}$ dia de armazenamento, o produto encontrava-se totalmente deteriorado, sem condições de comercialização e consumo.

PALAVRAS-CHAVE: atmosfera modificada, armazenamento, Brassica oleracea var. capitata.

\section{EFFECTS OF LOW DENSITY POLYETHYLENE PACKAGING AND TEMPERATURE IN THE CONSERVATION OF FRESH-CUT CABBAGE}

SUMMARY: This research studied the effect of low density polyethylene packaging and storage temperature on the preservation of fresh-cut (minimally processed) cabbage. The cabbages, previously cooled to a temperature of $10{ }^{\circ} \mathrm{C}$, were selected, washed, cut in four parts (with the central stalk removed), sanitized, cut in strips, rinsed, put in the centrifuge, weighed and stored in plastic packaging of low density polyethylene $(70 \mu \mathrm{m})$, and then stored in cold chambers at temperatures of 1 and $10^{\circ} \mathrm{C}$ for 20 days. The following aspects were evaluated: carbon dioxide, oxygen and ethylene in the internal atmosphere of the package as well as, $\mathrm{pH}$, titratable acidity, total soluble solids, vitamin $\mathrm{C}$, loss of fresh mass and the total soluble solids/acidity in the fresh-cut cabbage ratio. The experimental design was entirely casual, with three repetitions. The analysis parameters, except for the vitamin $\mathrm{C}$, loss of fresh mass and ethylene, presented significant variation between the temperatures and days of storage. The cabbage stored at a temperature of $1{ }^{\circ} \mathrm{C}$ presented a shelf life of around 15 days, significantly higher than that stored at $10^{\circ} \mathrm{C}$. At this temperature, on the $8^{\text {th }}$ day of storage, the product was completely decayed, unfit for commercialization or consumption.

KEYWORDS: modified atmosphere, storage, Brassica oleracea var. capitata.

\footnotetext{
${ }^{1}$ Eng. Agr., Mestre, Doutoranda em Engenharia Agrícola, FEAGRI/UNICAMP, Cidade Universitária Zeferino Vaz, Caixa Postal 6011, Campinas - SP. Fone: (0XX19) 3788.1075, Fax: (0XX19) 3788.1010, e-mail: mrinaldi@agr.unicamp.br.

${ }^{2}$ Prof. Dr., FEAGRI/UNICAMP, Cidade Universitária Zeferino Vaz, Caixa Postal 6011, Campinas - SP.

Recebido pelo Conselho Editorial em: 2-6-2003

Aprovado pelo Conselho Editorial em: 5-5-2004
} 


\section{INTRODUÇÃO}

A tecnologia de processamento mínimo apresenta dois propósitos: primeiro, manter o produto com características de fresco, além de fornecê-lo numa forma conveniente, sem perder sua qualidade nutricional; segundo, o produto deve apresentar uma vida de prateleira suficientemente longa para tornar sua distribuição possível aos potenciais consumidores (AHVENAINEN, 1996). As baixas temperaturas, quando associadas com atmosfera modificada durante o armazenamento, reduzem a ascensão respiratória e a síntese de etileno em hortaliças folhosas minimamente processadas, como alface (SINGH et al., 1972a, b) e o brócoli (BARTH et al., 1993) e também em frutos de maçã (KIM et al., 1993; NICOLI et al., 1994), melão, kiwi, mamão e abacaxi (O'CONNOR-SHAW et al., 1994) minimamente processados. Segundo SARANTÓPOULOS et al. (1996), a embalagem deve funcionar como a membrana que foi retirada com o processamento, reduzindo a perda de água, o ataque de microrganismos e outras reações. CANTWELL \& SUSLOW (1999) afirmam que a taxa respiratória de alface e repolho em tiras é 200-300\% maior que nesses produtos intactos e permanece alta durante todo o período de armazenamento. Essa taxa de respiração e deterioração pode ser minimizada pelo resfriamento rápido do produto e armazenamento a $5{ }^{\circ} \mathrm{C}$ ou menos.

KADER et al. (1998) afirmam que o mínimo de $\mathrm{O}_{2}$ tolerado pelo repolho na atmosfera da embalagem é de $2 \%$ e $5 \%$ de $\mathrm{CO}_{2}$, sendo que a faixa de temperatura recomendada para conservar o repolho é entre 0 e $5{ }^{\circ} \mathrm{C}$, e a concentração ideal de $\mathrm{O}_{2}$ e $\mathrm{CO}_{2}$ é, respectivamente, 2-3\% e 3-6\%. No entanto, GORNY (1997) recomenda também essa faixa de temperatura, sendo que $0{ }^{\circ} \mathrm{C}$ seria a temperatura ótima, e uma concentração de $\mathrm{O}_{2}$ entre 5-7,5\% e $15 \%$ de $\mathrm{CO}_{2}$, com exceção do repolho Chinês que deve ser armazenado com 5\% de $\mathrm{O}_{2}$ e $\mathrm{CO}_{2}$. A exposição de frutas e hortaliças frescas acima dos limites mínimos de $\mathrm{O}_{2}$ e máximos de $\mathrm{CO}_{2}$ pode provocar o aumento da respiração anaeróbica e o conseqüente acúmulo de etanol e acetaldeído, causando odores indesejáveis. Teores de $\mathrm{O}_{2}$ e $\mathrm{CO}_{2}$ acima dos limites tolerados pelo produto podem também induzir desordens fisiológicas, como o desenvolvimento de manchas marrons em alfaces, o escurecimento da superfície em aspargos e o escurecimento interno em repolhos (KADER et al., 1989 e CHITARRA \& CHITARRA, 1990).

Dessa forma, para obter um produto minimamente processado de boa qualidade, é fundamental mantê-lo sob refrigeração, com embalagem adequada a fim de prover a manutenção das características originais e estender a vida de prateleira do mesmo. Assim, procurou-se analisar o comportamento do repolho minimamente processado, acondicionado em embalagem de polietileno de baixa densidade mantida em duas condições de temperatura $\left(1\right.$ e $\left.10^{\circ} \mathrm{C}\right)$.

\section{MATERIAL E MÉTODOS}

Repolhos (Brassica oleracea var. capitata) dar cultivar Sagitarius, obtidos de produtor da região de Campinas - SP, foram resfriados à temperatura de $10^{\circ} \mathrm{C}$, selecionados e lavados em água corrente, cortados em quatro partes com a retirada do talo central, higienizados com solução contendo $150 \mathrm{mg} \mathrm{L}^{-1}$ de cloro ativo por $10 \mathrm{~min}$, fatiados em cortador industrial de vegetais, enxaguados com solução contendo $5 \mathrm{mg} \mathrm{L}^{-1}$ de cloro ativo e, posteriormente, centrifugados por $5 \mathrm{~min}$ em centrífuga doméstica $(800 \mathrm{x} \mathrm{g})$, pesados $(300 \mathrm{~g})$ e acondicionados em embalagens plásticas $(0,30 \times 0,24 \mathrm{~m}) \mathrm{de}$ polietileno de baixa densidade $(70 \mu \mathrm{m})$ sem perfurações. Após o fechamento das embalagens em seladora industrial, marca Selovac, modelo 200B, as mesmas foram armazenadas em câmaras frias, em duas condições de temperatura $\left(1\right.$ a $\left.10^{\circ} \mathrm{C}\right)$, por 20 dias. A cada cinco dias, avaliaram-se $\mathrm{pH}$, acidez titulável, sólidos solúveis totais, vitamina $\mathrm{C}$, perda de massa fresca e "ratio" (relação entre sólidos solúveis totais e acidez). A concentração de dióxido de carbono, oxigênio e etileno da atmosfera interna da embalagem foi analisada no primeiro, segundo, quinto, sétimo e oitavo dias de armazenamento. Com relação às temperaturas utilizadas, $1{ }^{\circ} \mathrm{C}$ representa a faixa de temperatura recomendada no armazenamento do repolho, sendo que $10^{\circ} \mathrm{C}$ está na faixa geralmente encontrada nas 
gôndolas dos supermercados. O delineamento estatístico foi inteiramente casualizado, com três repetições para cada tratamento ( 1 embalagem/2 temperaturas), em que cada repetição consistiu em uma amostra a ser analisada. As análises estatísticas foram realizadas com o auxílio do software ESTAT - Sistema para Análise Estatística - UNESP, Câmpus de Jaboticabal.

As concentrações de $\mathrm{CO}_{2}$ e $\mathrm{O}_{2}$ do espaço livre das embalagens foram determinadas em cromatógrafo a gás (Mod. CG 3400 Varian), equipado com detector de condutividade térmica e coluna empacotada com Chromosorb 106 (60-80 mesh, 1,8 m de comprimento e 3,2 mm de diâmetro interno). Utilizou-se como gás de arraste o hélio (He - $460 \mathrm{kPa}$ ), com o fluxo de 20-25 mL $\mathrm{min}^{-1}$. As temperaturas da coluna, do injetor e do detector foram, respectivamente, $40 ; 70$ e $150{ }^{\circ} \mathrm{C}$. A corrente utilizada foi de $138 \mathrm{~mA}$, com atenuação de $8 \mathrm{~mA}$. Esse mesmo cromatógrafo possui um detector de ionização de chama e coluna empacotada com Molisieve 5A 45/60 (80-100 mesh, 1,8 m de comprimento e 3,2 $\mathrm{mm}$ de diâmetro interno); as temperaturas da coluna, do injetor e do detector foram, respectivamente, $40 ; 70$ e $150{ }^{\circ} \mathrm{C}$. A pressão e o fluxo de $\mathrm{N}_{2}$ (gás de arraste), do ar sintético e do hidrogênio $\left(\mathrm{H}_{2}\right)$ foram, respectivamente, $280\left(20-25 \mathrm{~mL} \mathrm{~min}^{-1}\right), 550\left(300-350 \mathrm{~mL} \mathrm{~min}^{-1}\right)$ e $280 \mathrm{kPa}$ (37-39 $\left.\mathrm{mL} \mathrm{min}^{-1}\right)$ para mensurar as concentrações de etileno.

Esse método de determinação consiste na coleta de alíquotas de $1 \mathrm{~mL}$ de gás, utilizando uma seringa hermética própria para cromatografia, através de um septo de silicone colocado nas embalagens. As taxas de produção de $\mathrm{CO}_{2}, \mathrm{O}_{2}$ e etileno foram calculadas em função do tempo, e os padrões utilizados foram, respectivamente, $10.000 ; 80.000$ e $6,8 \mathrm{mg} \mathrm{L}^{-1}$.

As análises químicas, a perda de massa fresca e a "ratio" foram realizadas da seguinte forma:

- pH: determinado diretamente pela imersão do eletrodo do pHmetro (potenciômetro) digital Analyser na solução obtida pela diluição de $10 \mathrm{~g}$ de suco de tiras de repolho obtido em centrífuga doméstica, diluídos em $90 \mathrm{~mL}$ de água destilada, segundo procedimento descrito por CARVALHO et al. (1990);

- Acidez titulável: foi determinada de acordo com INSTITUTO ADOLFO LUTZ (1985): $10 \mathrm{~g}$ de suco obtidos de tiras de repolho centrifugados em centrífuga doméstica e diluídos em $90 \mathrm{~mL}$ de água destilada e posteriormente titulados com solução de hidróxido de sódio $(\mathrm{NaOH})$ a $0,01 \mathrm{~N}$ até $\mathrm{pH}$ 8,1. Os resultados expressos em porcentagem de ácido cítrico (que é o ácido presente em maior quantidade no repolho), ou seja, gramas de ácido cítrico/100 mL de produto (repolho);

- Sólidos solúveis totais (SST): o teor de sólidos solúveis totais foi determinado por meio da leitura em refratômetro manual, com precisão de 0,1 , do suco de tiras de repolho obtido pela centrifugação em centrífuga doméstica, segundo BOLIN \& HUXSOLL (1991). Os resultados foram expressos em ${ }^{\circ}$ Brix;

- Vitamina C: determinada segundo metodologia de CARVALHO et al. (1990), a qual se baseia na redução do indicador 2,6-diclorobenzenoindofenol (DCFI) pelo ácido ascórbico, sendo expresso em mg de ácido ascórbico/100 g de produto, e

- Perda de massa fresca: realizada utilizando-se de balanças digitais de precisão de $0,01 \mathrm{~g}$. O ensaio permitiu avaliar a perda de água durante a armazenagem frigorificada, e os resultados estão expressos em porcentagem, de acordo com a eq.(1):

$$
\frac{\text { Massa Inicial - Massa Final }}{\text { Massa Inicial }} 100
$$

- "Ratio": é a relação entre o teor de sólidos solúveis totais e a acidez titulável de um produto, que dá a indicação sobre o sabor e o estado de maturação. 


\section{RESULTADOS E DISCUSSÃO}

$\mathrm{O}$ filme de polietileno de baixa densidade $(70 \mu \mathrm{m})$, submetido à temperatura de $1^{\circ} \mathrm{C}$, apresentou tendência de estabilização na concentração de $\mathrm{CO}_{2}$ ao redor de $12 \%$, a partir de 48 horas de armazenamento, permanecendo praticamente constante até o $8^{\mathrm{o}}$ dia (Figura 1). No $12^{\mathrm{o}}$ dia, ocorreu um decréscimo na concentração desse gás no interior da embalagem, com posterior aumento até o $20^{\circ}$ dia. $\mathrm{O}$ menor teor de oxigênio foi encontrado no $5^{\mathrm{o}}$ dia de análise (em torno de 5\%). Para a temperatura de $10{ }^{\circ} \mathrm{C}$, essa mesma embalagem apresentou tendência de estabilização na concentração de $\mathrm{CO}_{2}$ (em torno de $20 \%$ ) a partir de 24 horas de armazenamento, permanecendo constante até 72 horas.

A partir do $3^{-0}$ dia, ocorreu um aumento significativo na concentração de $\mathrm{CO}_{2}$, chegando ao máximo valor de $27 \%$ no $7^{\circ}$ dia e uma tendência de diminuição nessa concentração observada no $8^{\underline{0}}$ dia, sendo que nessa data as amostras se encontravam totalmente deterioradas, ou seja, sem condições de comercialização e consumo. O oxigênio nessa temperatura apresentou queda brusca nas primeiras 24 horas de armazenamento, permanecendo praticamente constante (em torno de $1 \%$ ) até o $8^{\text {o }}$ dia de armazenamento. Dessa forma, pode-se concluir que a temperatura influenciou diretamente na atividade respiratória desse produto.

A maior concentração de $\mathrm{CO}_{2}$ apresentada pelas embalagens submetidas à temperatura de $10^{\circ} \mathrm{C}$ pode ter ocorrido pela influência da temperatura na taxa respiratória do produto e na propriedade de barreira ao $\mathrm{CO}_{2}$ do filme utilizado. De acordo com ZAGORY (1995), a taxa respiratória dos frutos e vegetais aumenta drasticamente com o aumento da temperatura. Isso também acontece com a permeabilidade do filme plástico. Entretanto, a respiração e a permeabilidade do filme não aumentam em taxas similares. Tipicamente, a taxa respiratória aumenta muito mais rápido que a permeabilidade do filme com o aumento da temperatura. Isso resulta, segundo esse autor, em uma importante limitação no uso de filmes para acondicionar produtos que respiram.

A atmosfera modificada deve ser utilizada para manter a qualidade e prolongar a vida útil dos produtos vegetais por meio da redução da atividade metabólica e principalmente da biossíntese e ação do etileno (KADER, 1986). No entanto, pelo método de determinação utilizado, não foi detectada produção de etileno no repolho minimamente processado.

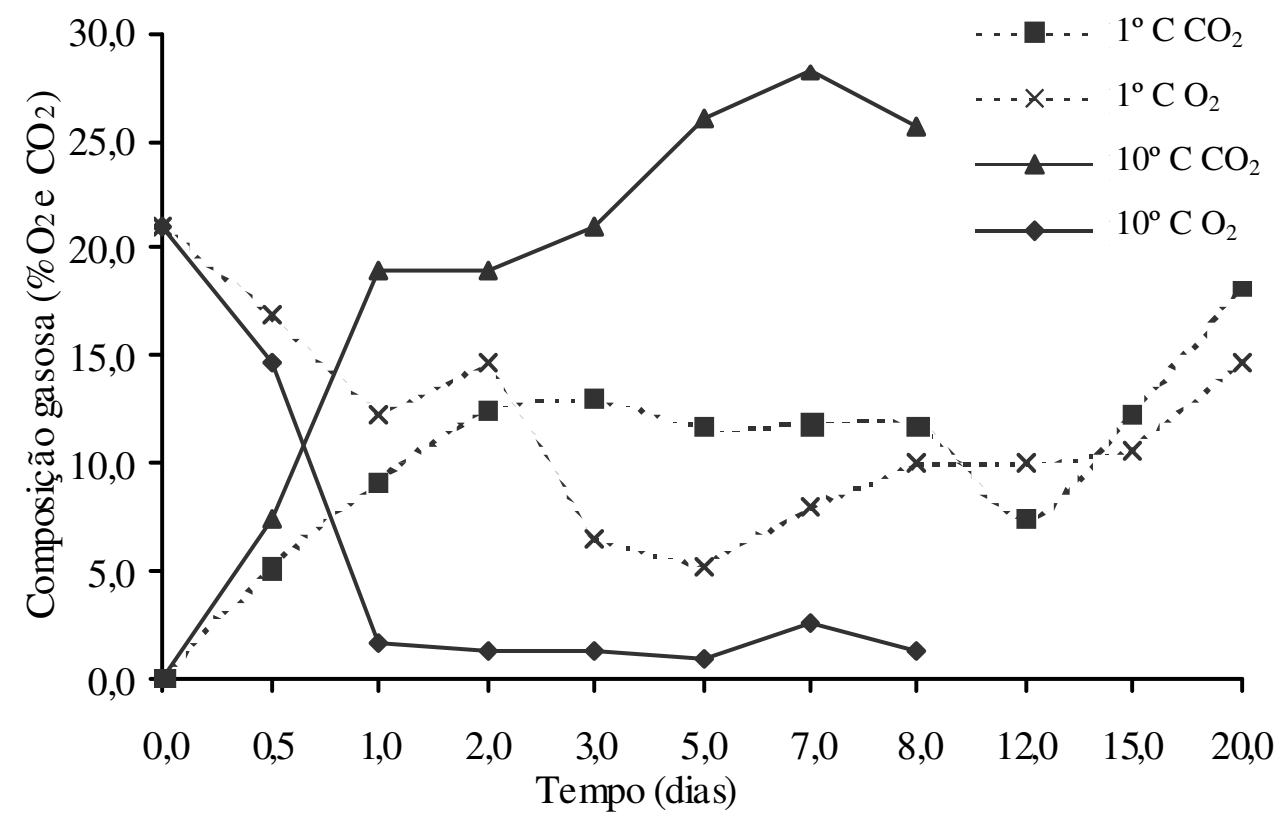

FIGURA 1. Composição gasosa da atmosfera interna de embalagens de polietileno de baixa densidade $(70 \mu \mathrm{m})$, contendo repolho minimamente processado, armazenado sob refrigeração. 
Quanto ao ácido ascórbico (Figura 2), nas duas temperaturas estudadas, não ocorreu diminuição significativa durante todo o período. Esse resultado contraria o observado por KLEIN (1987), de que o teor de ácido ascórbico pode ser menor nos produtos vegetais minimamente processados quando comparado ao dos produtos intactos. Isso porque se considera que os danos decorrentes do corte podem conduzir à perda desse ácido, uma vez que esse é suscetível à degradação quando em presença de luz e oxigênio. Segundo o mesmo autor, o ácido ascórbico pode ser oxidado por meio da interação com enzimas, como ascorbato oxidase, polifenoloxidase, citocromo oxidase e peroxidase, concluindo que o repolho, tanto intacto como minimamente processado, apresenta pequena perda dessa vitamina. No entanto, a perda de vitamina $\mathrm{C}$ pode variar de acordo com a espécie e condições de armazenamento, sendo que KLIEBER \& FRANKLIN (1999), ao estudar o conteúdo de ácido ascórbico em repolho Chinês minimamente processado, concluíram que, durante 11 dias de armazenamento a $4{ }^{\circ} \mathrm{C}$, houve diminuição significativa. BARTH \& ZHUANG (1996), avaliando brócoli minimamente processado acondicionado em embalagens plásticas sob atmosfera modificada, submetido a armazenamento refrigerado $\left(5^{\circ} \mathrm{C}\right)$, observaram que os teores de vitamina $\mathrm{C}$ se mantiveram praticamente estáveis na faixa de $5 \mathrm{mg} / 100 \mathrm{~g}$ de matéria seca, por um período de seis dias; entretanto, o armazenamento em embalagens perfuradas $(1 \mathrm{~cm}$ de diâmetro a cada $3 \mathrm{~cm})$ permitiu a redução da vitamina $\mathrm{C}$ para, aproximadamente, $2 \mathrm{mg} / 100 \mathrm{~g}$ de matéria seca, corroborando a hipótese de que a atmosfera modificada passiva é uma das ferramentas essenciais na manutenção da vida útil do produto.
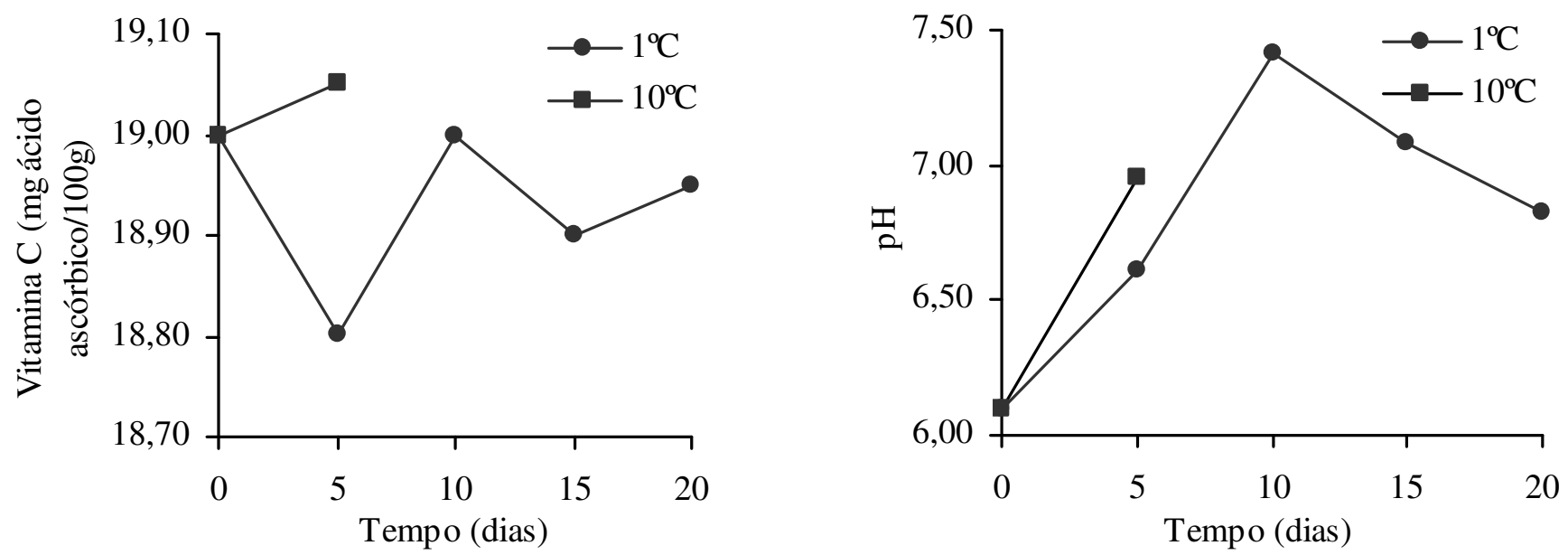

FIGURA 2. Vitamina $\mathrm{C}$ e $\mathrm{pH}$ em repolho minimamente processado, acondicionado em embalagens de polietileno de baixa densidade $(70 \mu \mathrm{m})$, armazenado sob refrigeração.

Na temperatura de $1{ }^{\circ} \mathrm{C}$, ocorreu aumento no $\mathrm{pH}$ (em torno de 1,3 unidade de $\mathrm{pH}$ ) até o $10^{\circ}$ dia de armazenamento, chegando a um valor máximo de 7,4, com posterior decréscimo até o $20^{\circ}$ dia (Figura 2). O repolho armazenado na temperatura de $10^{\circ} \mathrm{C}$ também apresentou aumento significativo até o $5^{\circ}$ dia de armazenamento, período em que o produto se encontrava em condições de consumo, diferindo significativamente dos valores obtidos na temperatura de $1{ }^{\circ} \mathrm{C}$. No entanto, RINALDI et al. (2002), ao estudarem repolho minimamente processado acondicionado em dois tipos de embalagens (filme de PVC e PET), mantidos nas temperaturas de $0 ; 5$ e $10{ }^{\circ} \mathrm{C}$ por 21 dias, concluíram que os repolhos armazenados na embalagem com filme de PVC não apresentaram diferenças significativas de $\mathrm{pH}$ nas três temperaturas analisadas, sendo que na embalagem PET, a variação foi significativa somente a $0{ }^{\circ} \mathrm{C}$, que apresentou menor $\mathrm{pH}$. SILVA (2000) não encontrou efeito significativo no $\mathrm{pH}$ de repolhos minimamente processados e embalados em filmes de polietileno de baixa densidade e polipropileno mantidos a $5 \pm 1{ }^{\circ} \mathrm{C}$ por sete dias. Segundo KADER (1986), o aumento de pH em 
vegetais minimamente processados pode estar relacionado com a resposta do tecido ao neutralizar a acidez gerada pelo $\mathrm{CO}_{2}$.

Observa-se, na Figura 3, que o comportamento da acidez foi semelhante para as duas temperaturas até o $5^{-}$dia de armazenamento, na qual ocorreu diminuição e diferença significativa entre as temperaturas. A $1{ }^{\circ} \mathrm{C}$, todos os valores obtidos de acidez titulável durante todo o experimento diferiram do $1^{\underline{o}}$ dia de análise, sendo que o $5^{\underline{o}}$ período (20 dias de armazenamento) diferiu do $2^{\underline{o}}$ e $4^{\underline{0}}$, que não diferiram entre si. A diminuição da acidez ocorrida em alguns períodos de análise pode ser justificada pelo consumo do próprio vegetal, na tentativa de se manter em seu estado inicial, pois, segundo KLUGE et al. (2002), os ácidos orgânicos são encontrados nos vacúolos das células na forma livre e combinados com sais, ésteres e glicosídeos, como fonte importante de energia para os vegetais, durante o processo de maturação, sendo que, nessa fase e no armazenamento, os mesmos sofrem oxidação no ciclo de Krebs.

Com relação aos valores de sólidos solúveis totais (Figura 3), observou-se diminuição durante o armazenamento, na temperatura de $1{ }^{\circ} \mathrm{C}$, a partir do $10^{\circ}$ dia de armazenamento, sendo que não houve diferença significativa entre o $15^{\circ}$ e o $20^{\circ}$ dia de armazenamento do repolho.
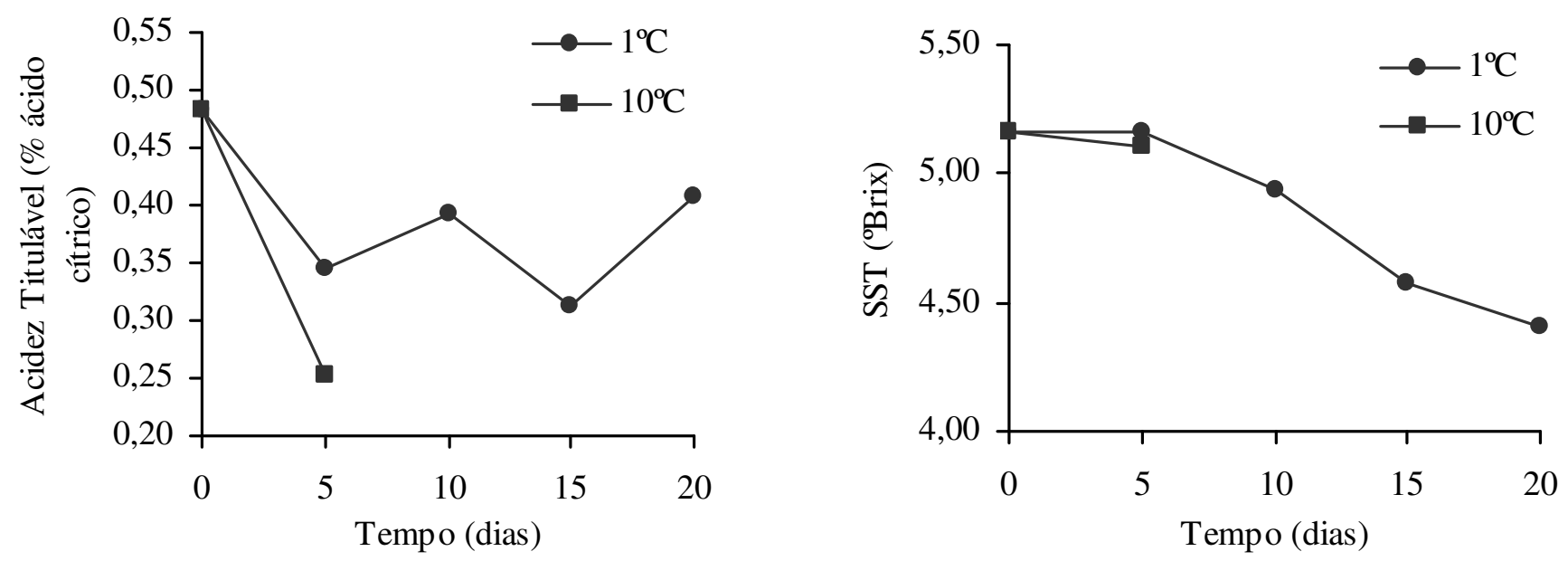

FIGURA 3. Acidez titulável e sólidos solúveis totais em repolho minimamente processado, acondicionado em embalagens de polietileno de baixa densidade $(70 \mu \mathrm{m})$, armazenado sob refrigeração.

Pode-se observar que a relação entre sólidos solúveis totais e acidez no repolho minimamente processado não apresentou comportamento linear na temperatura de $1{ }^{\circ} \mathrm{C}$, refletindo o comportamento dos componentes isoladamente (Figura 4). Nessa temperatura, a maior relação ocorreu no $5^{\circ}$ e $15^{\circ}$ dias de armazenamento. Para $10^{\circ} \mathrm{C}$, a maior relação, com o valor de 20 , ocorreu no $5^{\circ}$ dia de armazenagem, estando acima do balanceamento organoléptico equilibrado, que é considerado entre os valores de $12 \mathrm{e}$ 18 (CARVALHO et al., 1990).

Não ocorreu perda significativa de massa fresca no repolho minimamente processado durante todo o período de armazenamento, nas duas temperaturas estudadas (Figura 5). 


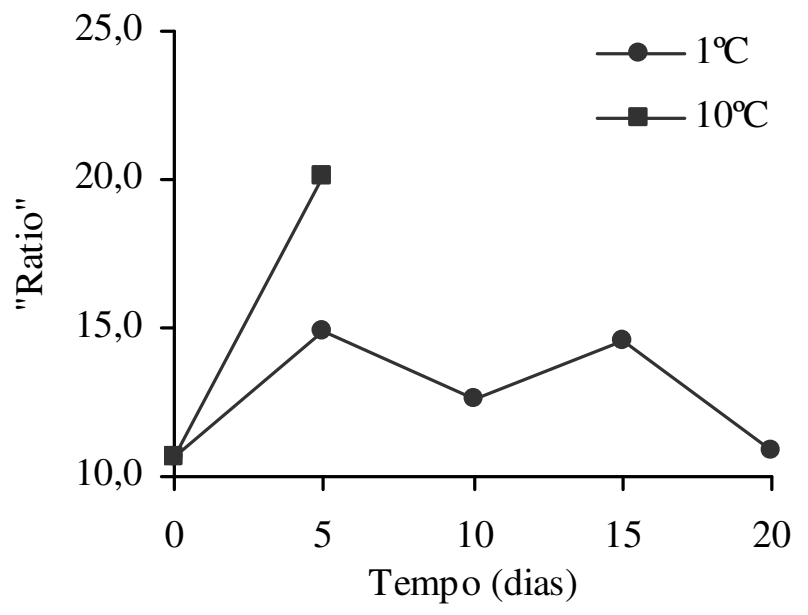

FIGURA 4. "Ratio" - relação entre sólidos solúveis totais e acidez titulável em repolho minimamente processado, acondicionado em embalagens de polietileno de baixa densidade $(70 \mu \mathrm{m})$, armazenado sob refrigeração.

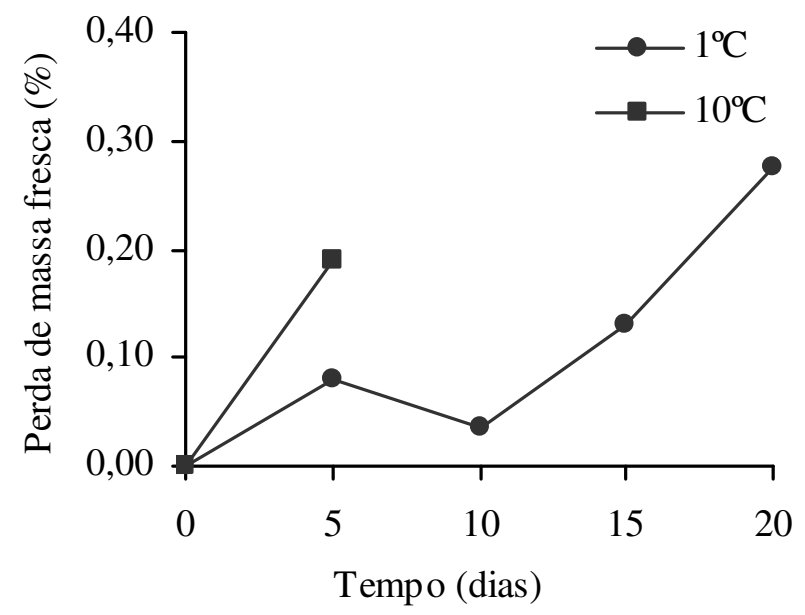

FIGURA 5. Perda de massa fresca em repolho minimamente processado, acondicionado em embalagens de polietileno de baixa densidade, $70 \mu \mathrm{m}$, armazenado sob refrigeração.

\section{CONCLUSÕES}

O repolho acondicionado em embalagem plástica de polietileno de baixa densidade $(70 \mu \mathrm{m})$, armazenado na temperatura de $1{ }^{\circ} \mathrm{C}$, apresentou vida de prateleira ao redor de 15 dias, sendo significativamente maior que a $10^{\circ} \mathrm{C}$. Nessa última, no $8^{\mathrm{o}}$ dia de armazenamento, o produto encontrava-se totalmente deteriorado, sem condições de comercialização e consumo.

A embalagem de polietileno de baixa densidade $(70 \mu \mathrm{m})$, armazenada à temperatura de $10^{\circ} \mathrm{C}$, ocasionou altos teores desse gás no interior da mesma, e o nível de $\mathrm{O}_{2}$ não foi suficiente para manter a respiração aeróbica mínima à manutenção do produto em condições de consumo, a partir do $5^{0}$ dia de armazenamento.

\section{AGRADECIMENTOS}

À Fundação de Amparo à Pesquisa do Estado de São Paulo e ao CPG/FEAGRI-UNICAMP, pelo auxílio concedido. 


\section{REFERÊNCIAS BIBLIOGRÁFICAS}

AHVENAINEN, R. New approaches in improving the shelf life of minimally processed fruit and vegetable. Trends in Food Science \& Technology, Cambridge, v.7, p.179-87, 1996.

BARTH, M.M.; KERBEL, E.L.; BROUSSARD, S.; SCHIMIDT, S.J. Modified atmosphere packaging protects market quality in broccoli spears under ambient temperature storage. Journal of Food Science, Chicago, v.58, p.1070-2, 1993.

BARTH, M.M.; ZHUANG, H. Packaging design affects antioxidant vitamin retention and quality of broccoli florets during postharvest storage. Post harvest Biology and Technology,Amsterdam, v.9, 141-50, 1996.

BOLIN, H.R.; HUXSOLL, C.C. Effect of preparation procedures and storage parameters on quality retention of salad-cut lettuce. Journal of Food Science, Chicago, v.56, n.1, p.60-7, 1991.

CANTWELL, M.; SUSLOW, T. Fresh-cut fruits and vegetables: aspects of physiology, preparation and handling that affect quality. In: ANNUAL WORKSHOP FRESH-CUT PRODUCTS: MAINTAINING QUALITY AND SAFETY, 5., 1999, Davis. Proceedings... Davis: University of California, 1999. Section 4b, p.1-22.

CARVALHO, C.R.L.; MANTOVANI, D.M.B.; CARVALHO, P.R.N.; MORAES, R.M.M. Análises químicas de alimentos. Campinas: ITAL, 1990. 121 p. (Manual Técnico).

CHITARRA, M.I.F.; CHITARRA, A.B. Pós-colheita de frutos e hortaliças: fisiologia e manuseio. Lavras: ESAL-FAEPE, 1990. 293 p.

GORNY, J.R. (Ed.) Fresh-cut fruits and vegetables and MAP. In: INTERNATIONAL CONTROLLED ATMOSPHERE RESEARCH CONFERENCE, 1997, Davis. Proceedings... v.5. 168 p. (Postharvest Horticultural Series, 19).

INSTITUTO ADOLFO LUTZ. Normas analíticas do Instituto Adolfo Lutz: métodos químicos e físicos para análise de alimentos. 2. ed. São Paulo: Instituto Adolfo Lutz, 1985. v.1, 371 p.

KADER, A.A. Potential applications of ionizing radiation in post harvest handling of fresh fruits and vegetables. Food Technology, Chicago, v.40, n.6, p.117-21, 1986.

KADER, A.A.; SINGH, R.P.; MANNAPPERUMA, J.D. Technologies to extend the refrigerated shelf life of fresh fruits and vegetables. In: TAUB, I.A.; SINGGH, R.P. (Ed.) Food storage stability. Boca Raton: CRC Press, 1998. p.419-34.

KADER, A.A.; ZAGORY, D.; KERBEL, E.L. Modified atmosphere packaging of fruit and vegetable. Critical Reviews in Food Science and Nutrition, Boca Raton, v.28, n.1, p.1-30, 1989.

KIM, D.M.; SMITH, N.L.; LEE, Y.C. Quality of minimally processed aple slices from selected cultivars. Journal of Food Science, Chicago, v.58, p.1115-17, 1993.

KLEIN, B.P. Nutritional consequences of minimal processing of fruits and vegetables. Journal of Food Quality, Wastport, v.10, p.179-93, 1987.

KLIEBER, A.; FRANKLIN, B. Ascorbic acid content of minimally processed chinese cabbage. Journal of the Science of Food and Agriculture, London, v.81, p.201-5, 1999.

KLUGE, A.R.; NACHTIGAL, J.C.; FACHINELLO, J.C.; BILHALVA, A.B. Fisiologia e manejo póscolheita de frutas de clima temperado. Pelotas: UFPEL, 2002. 214 p.

NICOLI, M.C.; ANESE, M.; SEVERINI, C. Combined effects in preventing enzymatic browing reactions in minimally processed fruit. Journal of Food Quality, Wastport, v.17, p.221-9, 1994.

O'CONNOR-SHAW, R.E.; ROBERTS, R.; FORD, A.L.; NOTTINGHAN, S.M. Shelf life of minimally processed honeydew, kiwifruit, papaya, pineaple and cantaloupe. Journal of Food Science, Chicago, v.59, p.1202-6, 1994. 
RINALDI, M.M.; BENEDETTI, B.C.; CALORE. L. Repolho minimamente processado: efeito da embalagem e temperatura de armazenamento. In: CONGRESSO BRASILEIRO DE ENGENHARIA AGRÍCOLA, 31., 2002, Salvador. Anais... Salvador: Sociedade Brasileira de Engenharia Agrícola, 2002. 1 CD ROM

SARANTÓPOULOS, C.I.G.L.; ALVES, R.V.; OLIVEIRA, L.M.; GOMES,T. Embalagens com atmosfera modificada. Campinas: CETEA/ITAL, 1996. 114 p.

SILVA, E.O. Fisiologia pós-colheita de repolho (Brassica oleracea cv. Capitata) minimamente processado. 2000. 90 f. Tese (Doutorado em Fisiologia Vegetal) - Universidade Federal de Viçosa, Viçosa - MG, 2000.

SINGH, B.; WANG, D.J.; SALUNKHE, D.K. Controlled atmosphere storage of lettuce. 1 - Effects on quality and respiration rate lettuce heads. Journal of Food Science, Chicago, v.37, p.48-51, 1972a.

SINGH, B.; WANG, D.J.; SALUNKHE, D.K. Controlled atmosphere storage of lettuce. 2 - Effects on biochemical composition of leaves. Journal of Food Science, Chicago, v.37, p.52-55, 1972 b.

ZAGORY, D. Principles and practice of modified atmosphere packaging of horticultural commodities. In: FARBER, J.M.; DODDS, S.K.L. (Ed.) Principles of modified-atmosphere and sous-vide product packaging. Lancaster, PA: Technomic Publishing, 1995. p.175-204. 